\title{
Deterministic inhomogeneous inertia ratchets
}

\author{
Shantu Saikia ${ }^{1,2}$, and Mangal C. Mahato ${ }^{*}$ \\ ${ }^{1}$ St.Anthony's College, Shillong-793001, India \\ ${ }^{2}$ Department of Physics, North-Eastern Hill University, Shillong-793022, India
}

\begin{abstract}
We study the deterministic dynamics of a periodically driven particle in the underdamped case in a spatially symmetric periodic potential. The system is subjected to a space-dependent friction coefficient, which is similarly periodic as the potential but with a phase difference. We observe that frictional inhomogeneity in a symmetric periodic potential mimics most of the qualitative features of deterministic dynamics in a homogeneous system with an asymmetric periodic potential. We point out the need of averaging over the initial phase of the external drive at small frictional inhomogeneity parameter values or analogously low potential asymmetry regimes in obtaining ratchet current. We also show that at low amplitudes of the drive, where ratchet current is not possible in the deterministic case, noise plays a significant role in realizing ratchet current.
\end{abstract}

Key words: Inhomogeneous systems, underdamped systems, deterministic ratchets, Langevin equation

PACS: 05.10.Gg, 05.40.-a, 05.40.Jc, 05.60.Cd

\section{Introduction}

Directed particle transport in asymmetric periodic (ratchetlike) potentials without the application of any obvious external bias but aided by thermal fluctuations has been widely studied both in overdamped and underdamped conditions $[1,2,3,4,5]$. This effect, called the ratchet effect, has also been obtained in underdamped symmetric periodic (non-ratchetlike) potential systems, by considering either the temperature 6, 7, 8,9] or the friction coefficient 10,11] to be space dependent. Ratchet current can also result in a symmetric periodic potential when the system is driven by an externally applied zero-mean

\footnotetext{
* Corresponding Author

Email address: mangal@nehu.ac.in (Mangal C. Mahato ${ }^{2}$ ).
} 
temporally asymmetric forcing aided by Gaussian white noise [12] or by the explicit application of nonthermal noise[13]14]. The ratchet effect is being studied intensively for about two decades and its applications envisaged in periodic systems that are common in many areas of natural sciences [15].

All the above ratchet models use the presence of (Gaussian or colored) noise (fluctuations) to obtain directed transport. In these models noise is essential. However, the study of deterministic ratchets, pioneered by Ref.[16], has also contributed significantly to the understanding of the subject. These deterministic ratchets, unaided by noise, are shown to yield current in overdamped[17]18], underdamped 14, 16, 19,20,21, 22, 23, 24, 25, 27, 28, as well as in Hamiltonian 29,30,31] periodic potential systems, and also in overdamped quenched disordered [32,34] systems. In these systems net current results, without the presence of applied nonzero average forcing or asymmetric fluctuations, due to the presence of various regular transporting or chaotic attractors depending on the initial conditions for given system parameter values. In Ref.[14] the symmetry criteria for the realization of ratchet current have been discussed in detail.

Owing to the complexity of the dynamics, for example the simultaneous presence of periodic and chaotic attractors, there is a need for proper choice of initial conditions and ensemble averaging over them to obtain realistic averages 14, 19, 29]. This fact has been exemplified in an (zero mean) ac modulated periodic Hamiltonian system 31]. It has been shown there that slower modulations of the symmetric periodic potential lead to asymmetric access (in momentum) of chaotic regimes in the phase space resulting in a giant net particle transport in one direction. The direction and magnitude of the current depends on the initial phase of the modulating ac drive. Of course, the net current must disappear if averaged over the initial phase.

The driven deterministic systems show frequent ensemble-averaged current reversals as the drive parameters are changed. Mateos identified bifurcation from a chaotic to a periodic regime as the mechanism for the average-current reversals [20] in these systems. However, the change in direction of individual single particle trajectories could be related to phase locking phenomena 21] due to the presence of various velocity attractors [24]. The average current directions are thus sensitive to initial conditions [38] which allow to explore these attractors selectively in the phase space. In a recent further investigation[19] it has been conjectured that, in general, bifurcations from chaos to periodic behaviour could be associated with abrupt changes (and not necessarily reversals) in the ensemble averaged currents.

In Ref. 34], the effect of different control parameters were analysed in the inertial limit. It was found that the control mechanisms were associated with the fractal nature of the basins of attraction of the mean velocity attractors and that small perturbations of the control variable could produce drift reversals. 
However, the effect of the presence of a weak subharmonic component in the ac drive field on the phase locked dynamics of a ratchet is to suppress chaos and stabilize regular orbits over large range of driving amplitudes [37].

The transport properties of inertial systems depend sensitively on the (constant) friction parameter[26,27,28]. The addition of noise to the system in the low damping regime makes the system dynamics robust against initial conditions [27]. Moreover, in a well defined damping window the efficiency of ratchet current generation becomes appreciable even for small applied periodic field amplitudes. In Ref. [28] the effect of noise on individual transporting deterministic trajectories is analysed. A small noise seem to have little effect on some trajectories in one direction whereas it destroys trajectories in the opposite direction resulting in a robust finite ratchet current.

In most of the above works (barring a few, for instance[14,29,31]), particle motion in periodic but asymmetric potentials is considered; for example a system with potential $V(x)=V_{0}(\cos x+b \cos 2 x)$ driven by a periodic field, $F(t)=a \cos \left(\omega t+\phi_{0}\right)$ in uniform friction media. In these studies the potential asymmetry $(b \neq 0)$ is the primary cause of net particle drift. In the present work, we consider a symmetric periodic potential, for example $V(x)=-V_{0} \sin x$, but in the presence of a space dependent friction coefficient $\gamma(x)=\gamma_{0}(1-\lambda \sin (x+\phi))$ (as in [10]). A massive charged particle moving in a periodic ionic lattice and in a medium with material density (friction) profile created by a stationary pressure wave can be thought of as a mechanical illustration of such systems 10,11. However, such systems have been studied earlier in a resistively and capacitatively shunted junction circuit model of small Josephson junctions 39,40]. The frictional inhomogeneity term emulating there the " $\cos \theta$ " term representing the coupling between the quasiparticle tunneling and Cooper pair tunneling across the Josephson junction. In Ref. [39] the phase space trajectories of this system are presented in detail.

In the present work we show that all the features of deterministic inertia ratchets can be observed even in the symmetric periodic potential system in an inhomogeneous medium. The frictional nonuniformity of the medium with phase shift $\phi(\neq 0, \pi)$ essentially emulates the effect of the asymmetry of the potential considered in earlier cases. Moreover, we show in our present inhomogeneous dissipative system that, for small inhomogeneity (roughly $\lambda \leq 0.6$ ), in order to get sensible ratchet current one also needs to average over the initial phase $\phi_{0}$ of the drive as in the Hamiltonian system[29]31]. Though ratchet current is a steady state $(t \rightarrow \infty)$ phenomenon one is likely to obtain a finite ratchet current, an obvious erroneous result, even at zero potential asymmetry in a uniform medium without this averaging. Interestingly, the bifurcation diagrams with and without the frictional inhomogeneity are qualitatively indistinguishable. However, the stroboscopic Poincaré plots differ qualitatively in the driven system as the inhomogeneity parameter $\lambda$ is varied. Links Vi 
The presence of noise (which is ubiquitous in natural environments) smooths out the current fluctuations so numerously present in the deterministic case [28,41]. In Ref. [41] a matrix continued fraction method is presented to obtain ratchet current in noisy periodically driven inertial systems. We further show in our inhomogeneous system that at low drive amplitudes noise plays a crucial role in getting ratchet (mean) current, that is conspicuously absent in the deterministic drive case, though at larger drive amplitudes one obtains ratchet current without the presence of noise.

\section{The model}

As mentioned earlier, in this work, we consider the motion of a particle in a periodic potential $V(x)=-V_{0} \sin (k x)$ which is symmetric in space (about $k x=(2 n+1) \pi / 2, n=0, \pm 1, \pm 2, \ldots)$. The friction coefficient $\gamma(x)=\gamma_{0}(1-$ $\lambda \sin (k x+\phi))$ is periodic with the same periodicity as the potential but has a phase difference. The system is driven periodically by an external periodic forcing $F(t)=a \cos \left(\omega t+\phi_{0}\right)$. We study the system in the deterministic regime i.e. in the absence of noise and also at the end in the presence of noise.

The one dimensional equation of motion of a particle of mass $m$ is given by the Langevin equation,

$$
m \frac{d^{2} x}{d t^{2}}=-\gamma(x) \frac{d x}{d t}-\frac{\partial V(x)}{\partial x}+F(t)
$$

The corresponding equation of motion in the presence of noise is given by

$$
m \frac{d^{2} x}{d t^{2}}=-\gamma(x) \frac{d x}{d t}-\frac{\partial V(x)}{\partial x}+F(t)+\sqrt{\gamma(x) T} \xi(t)
$$

In Eq.2, $T$ is the temperature in units of the Boltzmann constant $k_{B}$. The Gaussian distributed fluctuating forces $\xi(t)$ satisfy the statistics: $\langle\xi(t)>=0$, and $<\xi(t) \xi\left(t^{\prime}\right)>=2 \delta\left(t-t^{\prime}\right)$. For convenience, we write down Eq.1 and Eq.2 in dimensionless units by setting $m=1, V_{0}=1, k=1$. In Eq.2, $T=2$ then corresponds to an energy equivalent equal to the potential barrier height at $a=0$. In terms of the reduced variables denoted again now by the same symbols, the two equations can be written as

$$
\frac{d^{2} x}{d t^{2}}=-\gamma(x) \frac{d x}{d t}+\cos x+F(t)
$$

and

$$
\frac{d^{2} x}{d t^{2}}=-\gamma(x) \frac{d x}{d t}+\cos x+F(t)+\sqrt{\gamma(x) T} \xi(t),
$$

where $\gamma(x)=\gamma_{0}(1-\lambda \sin (x+\phi))$. Thus the periodicity of the potential $V(x)$

and also the friction coefficient $\gamma$ is $2 \pi$. The potential barrier between any 
two consecutive wells of $V(x)$ persists for all field amplitudes $a,(0<a<1)$ and it just disappears at the critical field value $a=a_{c}=1$. Notice that for $\phi=n \pi, n=0, \pm 1, \pm 2, \ldots$ the deterministic Eq. 3 should yield exactly the same trajectory but in the opposite direction on letting $x \rightarrow x-\pi / 2$ and $t \rightarrow-t$ and in the presence of a thermal noise Eq.4 should give the same ensemble averaged current but again in the opposite direction[14]. Thus in order that a ratchet current appears the symmetry of the system must be broken using $\phi \neq n \pi$.

The noise variable, $\xi$ in Eq.4, satisfies exactly similar statistics as earlier. Eq.3 and Eq.4 are solved numerically using the Heun's method for solving differential equations. The particle trajectories are obtained for given initial conditions for fixed values of $a$ and $\gamma_{0}$. Also, the steady state mean velocity of the particles are calculated.

For the deterministic case the average velocity for a single particle (with a given initial condition) is obtained by the relation [24]

$$
\langle v\rangle=\frac{x\left(t_{\max }\right)-x\left(t_{\text {tran }}\right)}{\left(t_{\max }-t_{\text {tran }}\right)},
$$

where $t_{\max }$ is the maximum time for which the particle is allowed to evolve and $t_{\text {tran }}$ is the duration of initial transients that we remove.

Also the mean velocity averaged over an ensemble of $N$ particles is defined as

$$
\langle\langle v\rangle\rangle=\frac{1}{N} \sum_{i=1}^{N}\langle v\rangle_{i},
$$

For the particle trajectory in the presence of noise, the steady state mean velocity $\bar{v}$ of the particle is obtained as

$$
\bar{v}=\left\langle\lim _{t \rightarrow \infty} \frac{x(t)}{t}\right\rangle
$$

where the average $\langle\ldots\rangle$ is evaluated over a large number of trajectories.

\section{$3 \quad$ Numerical Results}

The phase space trajectories of the deterministic Eq.3 was investigated earlier in Ref. [39]. Also, for symmetric systems $(\lambda=0$, in Eq.3) the regimes of chaotic (nontransporting) and periodic motion were given in detail in Ref. [42. In the present work the particle is allowed to evolve for a long time so that the system reaches a steady state condition to investigate ratchet 


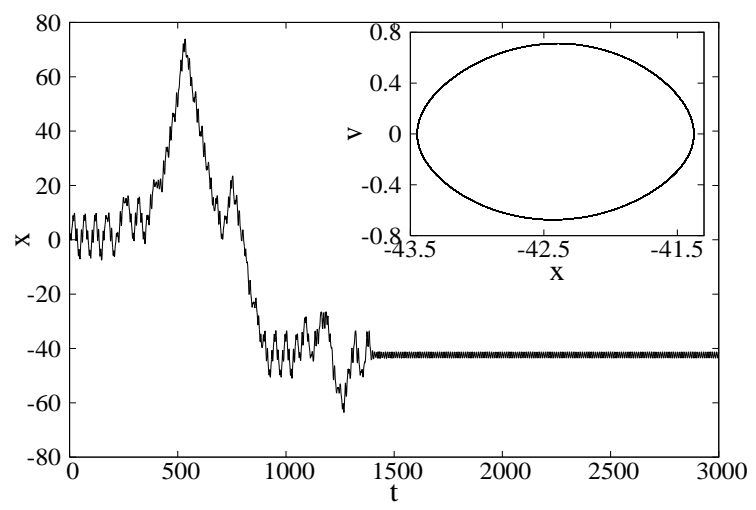

Fig. 1. Plot of particle trajectory for $a=0.5$ and $\tau=10$ (main figure) and the corresponding phase space trajectory; $x(0)=\pi / 2, v(0)=0, \gamma_{0}=0.12, \phi=0.35$, $\lambda=0.9$.

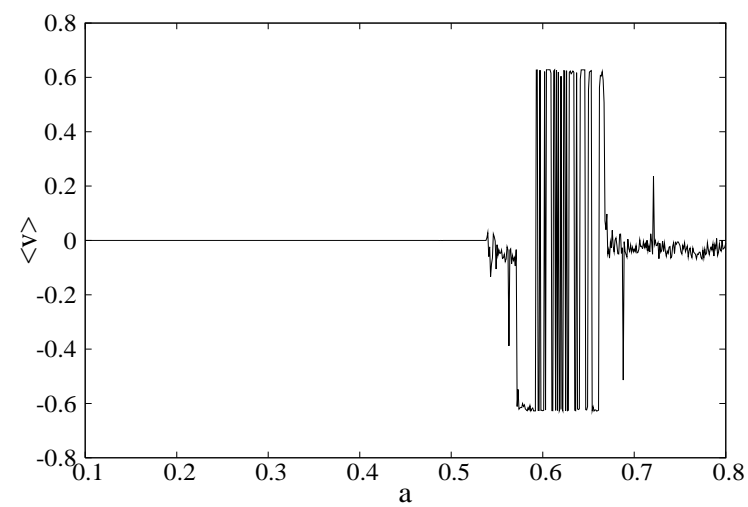

Fig. 2. Variation of mean velocity $\langle v\rangle$ with amplitude $a$ for a single particle; $v(0)=0$, $x(0)=\pi / 2, \gamma_{0}=0.12, \phi=0.35, \lambda=0.9$.

effect. We solve Eq.3 (for the deterministic case) and Eq.4 (when noise is included) using the second order Heun's method with time step size $\Delta t=$ 0.001. For the calculation of deterministic average velocity (Eq.5) we take $t_{\text {max }}=10^{6}$ and $t_{\text {tran }} \approx 10^{4}$. Throughout our work, unless otherwise stated, we keep $\lambda=0.9, \gamma_{0}=0.12$ and $\phi=0.35$. We partly repeat the investigations done earlier [20,21,24] replacing the asymmetric periodic potentials by a symmetric periodic potential but instead of a uniform-friction-medium we consider here a periodically varying friction coefficient and present our results below.

\subsection{Phase locked dynamics and sensitivity to initial conditions}

The dynamics of the particle is critically dependent on the amplitude $a$ and frequency $\omega$ of the zero-mean external periodic drive 34]. The dissipative system goes to the only fixed point attractor at $v=0, x=(2 n-1) \pi / 2$ when the system is not driven $(a=0)$. For a given (non-zero) amplitude and frequency of the drive the individual particle trajectories depend sensitively on the initial conditions. In the low amplitude regime (below a particular critical amplitude 


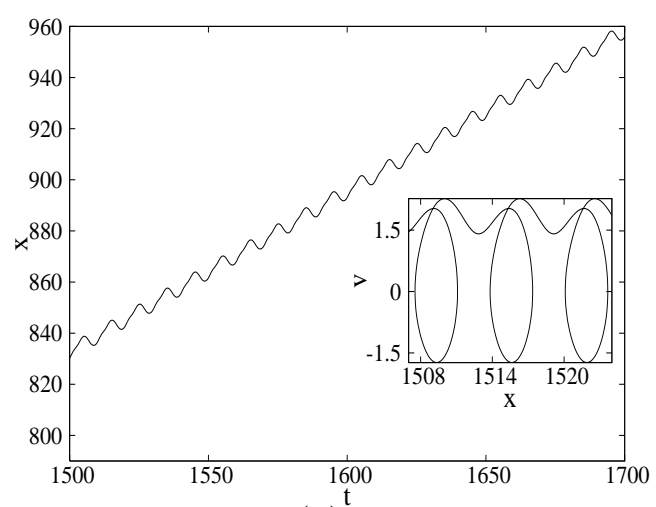

(a)

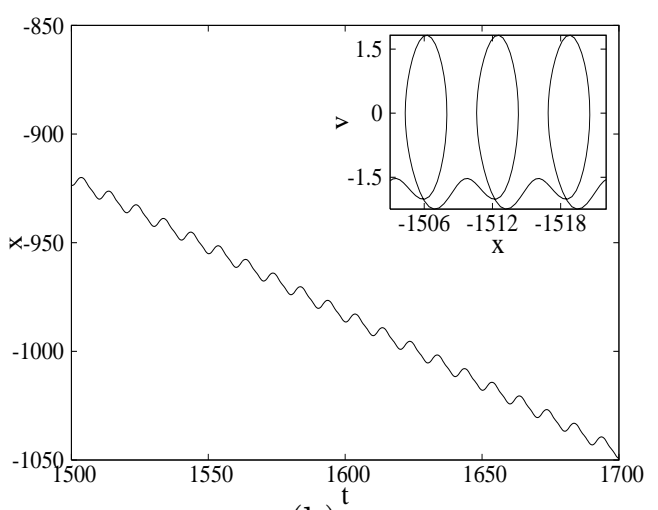

(b)

Fig. 3. Particle trajectories (main figures) and the corresponding phase space plots (insets) showing different locking behaviour of the particle. For $a=0.5931$ (fig. a), the particle gets locked in the positive sense while for $a=0.592$ (fig. b), the particle gets locked in the negative sense. $\tau=10, x(0)=\pi / 2, v(0)=0$ for both cases.

depending on the frequency) the particle, after initial transients, gets invariably trapped to a periodic attractor in some potential minima $x=(2 n+1) \pi / 2$ $(n=0, \pm 1, \pm 2, \ldots)$ and executes periodic motion within the potential well (Fig.1). On the average these cases give zero net current as is evident from the phase space trajectory (Inset, Fig.1). Only beyond certain amplitude $a$ (for a given initial condition and drive frequency $\omega$ ), the particle starts evolving giving rise to either periodic or chaotic dynamics.

Fig.2 shows the mean velocity $\langle v\rangle$ for a typical single particle trajectory (with the same initial condition $(x(0)=\pi / 2$ and $v(0)=0$ ) as a function of drive amplitude $a$ and period $\tau=10$, where $\tau=2 \pi / \omega$. There are numerous changes in the direction of current as the amplitude $a$ is set at different values. For example, whereas $a=0.5931$ gives a positive $\langle v\rangle, a=0.592$ for the same initial conditions gives mean velocity $-\langle v\rangle$. This behaviour can be traced to amplitude dependent phase locked periodic behaviour of particle motion as shown in Figs.3a,b. As the border between the basins of these attractors are crossed with the variation of the amplitude of the drive the single particle shows a change in the direction of $\langle v\rangle[21,24]$.

Also, the sensitivity of the trajectories to initial conditions can be inferred by the following observation. For $a=0.7$ and $\tau=10$, the intial condition $v(0)=$ $0, x(0)=1.38309$ gives a transporting phase locked trajectory in the positive direction as in Fig. 3a, whereas $v(0)=0, x(0)=1.383093$ gives locked periodic nontransporting trajectory as in Fig. 1 and $v(0)=0, x(0)=1.38309704$ results in a transporting phase locked trajectory in the negative direction as in Fig. 3b. The sensitivity of the initial conditions demands that meaningful net particle velocity for a given $a$ and $\omega$ be obtained by averaging over a large number of particle trajectories corresponding to various initial conditions. 
As the nature of the particle trajectory depends critically on the chosen initial condition, for realistic averages we calculate the velocity (Eq.6), averaged over an ensemble of $N$ (typically equal to 500) particles. The particles are taken with identical initial velocities $\left(v_{i n i}=0\right)$, but with initial positions uniformly distributed between two consecutive potential maxima 20]. The particle trajectories, and also the phase space diagrams, are studied to reveal the characteristics of the particle dynamics. The bifurcation diagrams are obtained by recording the values of $\dot{x}\left(t_{p}\right)$ at times $t_{p}=n_{p} \tau$, for each value of the control parameter (the amplitude of the external drive), where $\tau=2 \pi / \omega$ is the period of the external drive and $n_{p}$ is an integer.

\subsection{Ratchet current and bifurcation diagrams}

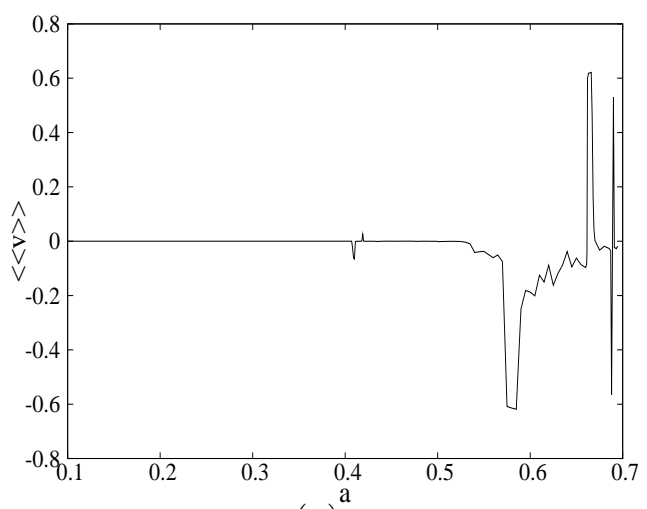

(a)

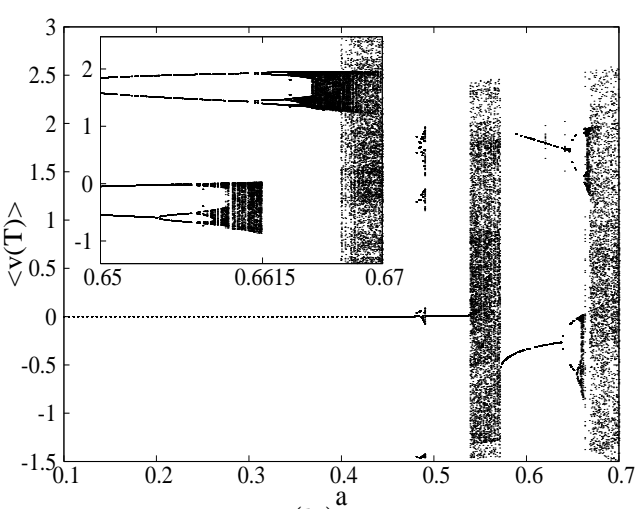

(b)

Fig. 4. Variation of ensemble averaged velocity $\langle\langle v\rangle\rangle$ with amplitude of drive $a$ (a). Fig.4b. shows the corresponding bifurcation diagram. Inset of (b) shows the amplitude at which the current reversal occurs in Fig.4a. $\tau=10, \gamma=0.12, \lambda=0.9$, $\phi=0.35$.

On ensemble averaging, ratchet currents (Fig.4a) are obtained in the deterministic inertial inhomogeneous system with a symmetric and periodic potential. The averaging is done with the same set of parameters as in Fig.2. The system shows current reversals as a function of the amplitude of the drive. However, most of the single particle current reversals appearing in Fig.2 disappear. This is due to the fact that different initial conditions select different attractors for a particular amplitude, all the other parameters remaining the same. So, at a particular amplitude of drive, some particles of the ensemble may move in the positive direction and some may move in the negative direction with different multiples of the fundamental locking velocity $v_{\omega}$ [21]. Others may show a chaotic dynamics giving rise to either zero velocity or some non-zero velocity on an average. The corresponding bifurcation diagram (Fig.4b) reflects this argument.

Fig.4b shows that the particle exhibits regimes of periodic and chaotic dynam- 


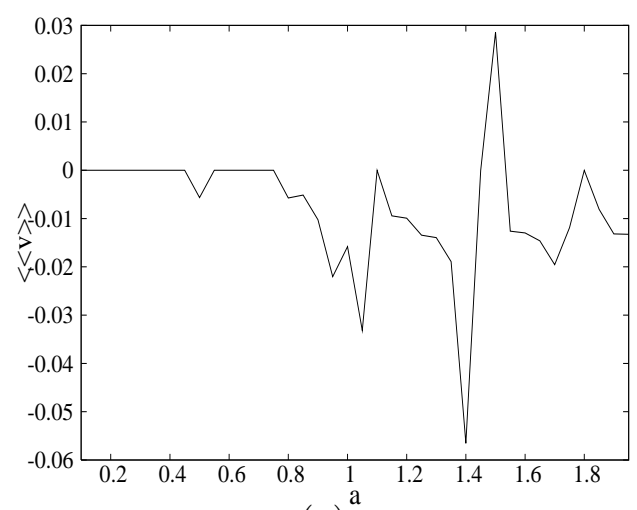

(a)

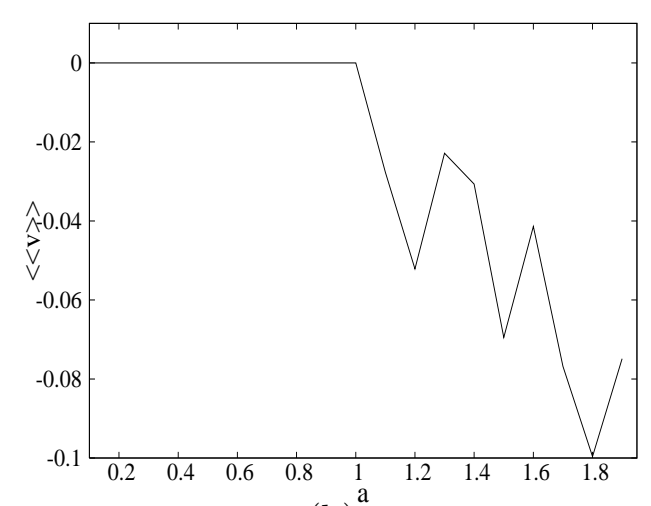

(b)

Fig. 5. Variation of ensemble averaged velocity $\langle\langle v\rangle\rangle$ with amplitude of drive $a$ for $\tau=100$ (a) and $\tau=1000(\mathrm{~b}) ; \gamma=0.12, \lambda=0.9, \phi=0.35$.

ics as a function of the control parameter $a$. The current reversal at $a=0.6615$ in Fig.4a is due to a bifurcation from a chaotic to a periodic regime (inset of Fig.4b ) which agrees with Mateos conjencture [20]. However, there could be many such bifurcations but without an accompanying current reversal[19].

\subsection{Frequency dependence of particle dynamics}

The nature of the particle dynamics i.e. the nature of the current and its reversals changes with the driving frequency $\omega=2 \pi / \tau$. A comparison between the current profile for three periods $\tau=10$ (Fig.4), $\tau=100$ and $\tau=1000$ (Fig.5) reflects this fact. For different frequencies, particle current starts developing at different amplitudes. This is because the amplitude of the drive at which the particle starts evolving out of a single-well trapped-state differs with frequency. For very low frequencies of the drive $\tau=1000$ (Fig.5b), particle currents are obtained only beyond the critical tilt ( $a=1.0$ for our case) whereas for $\tau=10$ current starts developing essentially around $a=0.55$. Also, whereas for the slower drive $\tau=1000$ there is no current reversal in the range of drive amplitudes shown, for $\tau=10$ and 100 there are several reversals of the direction of current in the same range of $a$. Thus, the mean velocity is expected to show a very complex behaviour in the $(a-\omega)$ space 34 .

\subsection{Role of frictional inhomogeneity and dependence on initial driving phase}

The particle dynamics in the present dissipative system with different degrees of frictional inhomogeneity $(\lambda)$, for $\phi=0.35$, starting with $\lambda=0$ is investigated. We note that $\lambda$ in the present work plays a role analogous to the potential asymmetry in the uniform friction case. Surprisingly, at certain drive amplitudes, even with zero inhomogeneity, non-zero (steady state, $t \rightarrow \infty$ ) 


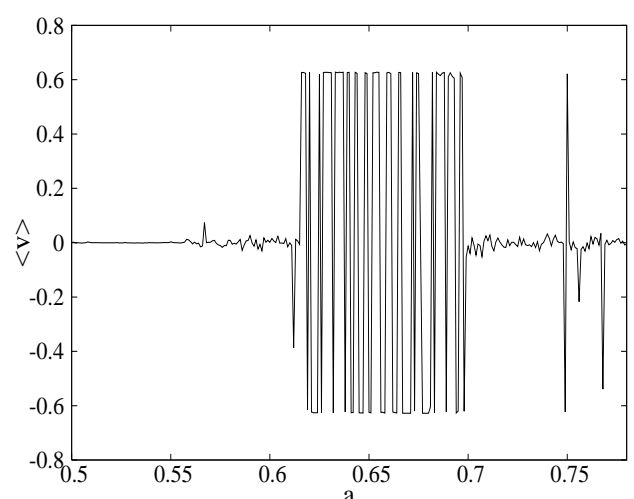

$(\mathrm{a})^{\mathrm{a}}$

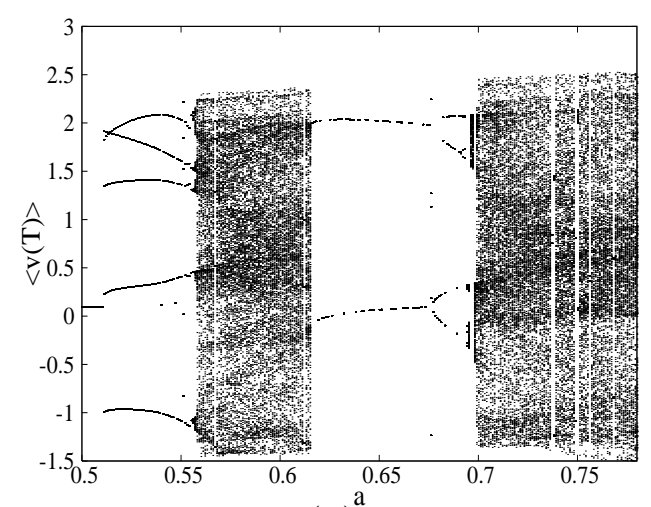

$(\mathrm{b})^{\mathrm{a}}$

Fig. 6. $\langle v\rangle$ versus $a$ plot for a single particle with $\lambda=0$ (a) and the corresponding bifurcation diagram (b). $\tau=10, x(0)=\pi / 2, v(0)=0$.

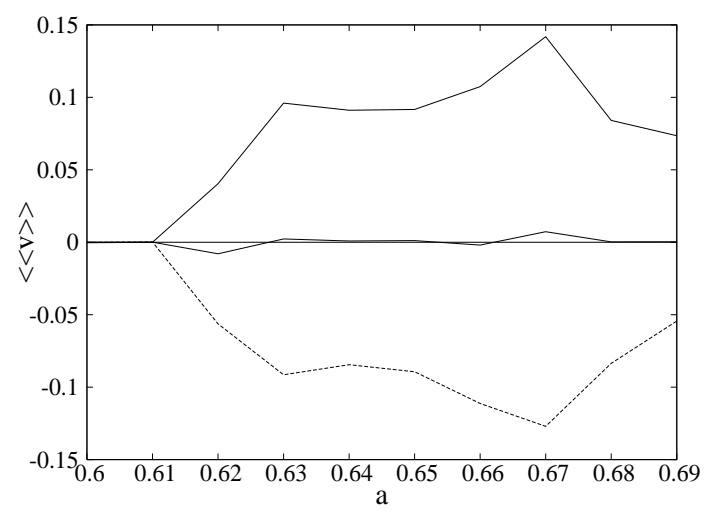

Fig. 7. Plot of $\langle\langle v\rangle\rangle$ versus $a$ with zero inhomogeniety $(\lambda=0)$ for initial phase of the external drive $\phi_{0}=0$ (lower dotted line) and $\phi_{0}=\pi$ (upper solid line). Middle line shows $\langle\langle v\rangle\rangle$ after averaging over initial phases. Zeroline is drawn for reference. $\tau=10, \lambda=0.9, \gamma=0.12, \phi=0.35$.

current is obtained, both at the single realisation (Fig.6) case, namely $\langle v\rangle$, as well as when averaged over a large number of realizations, $\langle\langle v\rangle\rangle$ (Fig.7). Instead, one would have expected the dissipative system to lose the initial memory by the time the transients get settled down. The velocities $\langle v\rangle$ wildly change with drive amplitude (Fig. 6(a)) but are finite. $\langle\langle v\rangle\rangle$ are not only finite but they vary smoothly with drive amplitude. This is obviously an erroneous result. Moreover, the bifurcation diagrams with inhomogeneity (Fig. 4(b)) and without inhomogeneity (Fig.6(b)) on comparison appear very similar and do not provide any clue to the error. The error could only come from lack of averaging over the initial phase $\phi_{0}$ of the zero-mean external periodic drive $F(t)$. The relevance of averaging over initial phase has earlier been discussed for Hamiltonian systems [29,31].

In Fig.7, the ensemble averaged current $\langle\langle v\rangle\rangle$ are shown for two values of the initial phase $\phi_{0}=0$ and $\pi$ of the external drive $F(t)$ of period $\tau=10$. Clearly, the initial bias due to a fixed value of $\phi_{0}$ determines the steady state current. 


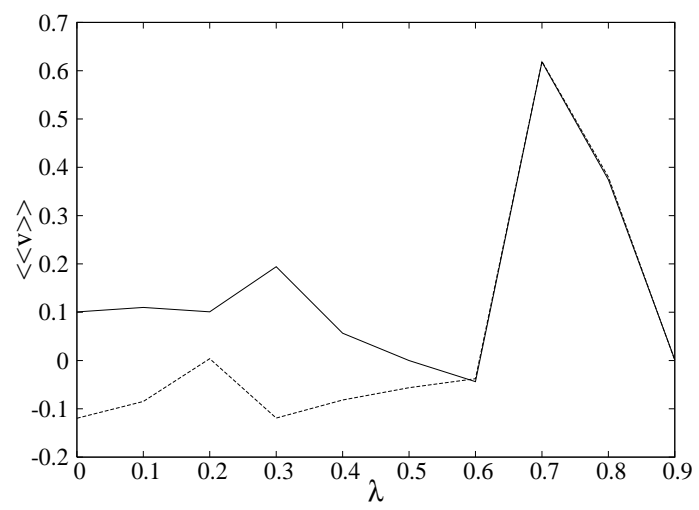

Fig. 8. Variation of $\langle\langle v\rangle\rangle$ with the asymmetry parameter $\lambda$ for initial phase of the external drive $\phi_{0}=0$ (lower dotted line) and $\phi_{0}=\pi$ (upper solid line); $\tau=10$, $a=0.67, \gamma=0.12, \phi=0.35$.

In order to get a sensible ratchet current one needs, therefore, to average over the initial phase $\phi_{0}$ too. The figure also indicates that averaging should be carried out over at least two values of $\phi_{0}$ which differ by $\pi$.

Interestingly, this averaging is required only for small inhomogeneities (Fig.8). For large asymmetries $\langle\langle v\rangle\rangle$ does not seem to depend on the initial phase $\phi_{0}$. It turns out that the critical inhomogeneity $\lambda=\lambda_{c} \approx 0.6$ (Fig.8). In the case of uniform friction the critical potential-asymmetry $b \approx 0.25$ which is what has been used incidentally by Mateos 20] and others corresponding to their parameter values. As the value of $\lambda$ is changed, the nature of the particle trajectories changes qualitatively.

In Fig.9 we present the typical trajectories $x(t)$ along with the stroboscopic phase points at regular time intervals $t=n \tau, n=1,2,3,$. for various values of the inhomogeneity parameter $\lambda$ for amplitude $a=0.67$, period $\tau=10$ and initial phase $\phi_{0}=0$ of the external field $F(t)$. For $\lambda=0.9$ all the trajectories are chaotic but nontransporting giving zero ratchet current. For $\lambda=0.8$ the trajectories are chaotic consisting of longer quasiperiodic transporting trajectories in the positive direction but interrupted by shorter similar trajectories in the negative direction giving finite ratchet current in the positive direction. For $\lambda=0.7$ the current $\langle\langle v\rangle\rangle$ is close to the maximum $(=2 \pi / \tau)$ corresponding to the fundamental velocity. In this case all the trajectories are quasiperiodic transporting in the same positive direction. The corresponding stroboscopic plot shows their chaotic nature. As the $\lambda$ is lowered from 0.7 to 0.6 we obtain a mixture of transporting quasiperiodic trajectories and transporting regular periodic trajectories. However, as the value $\lambda=0.6$ is reached their directions of average motion gradually become equal giving zero current at $\lambda=0.6$. As mentioned earlier, $\langle\langle v\rangle\rangle$ is independent of $\phi_{0}$ for the considered parameters. For values of $\lambda \leq 0.5$ all the trajectories are transporting regular periodic with same average slope but differing in direction. For $\phi_{0}=0$ there are more negative-slope trajectories and vice-versa for $\phi_{0}=\pi$. The five-paneled Fig.9 


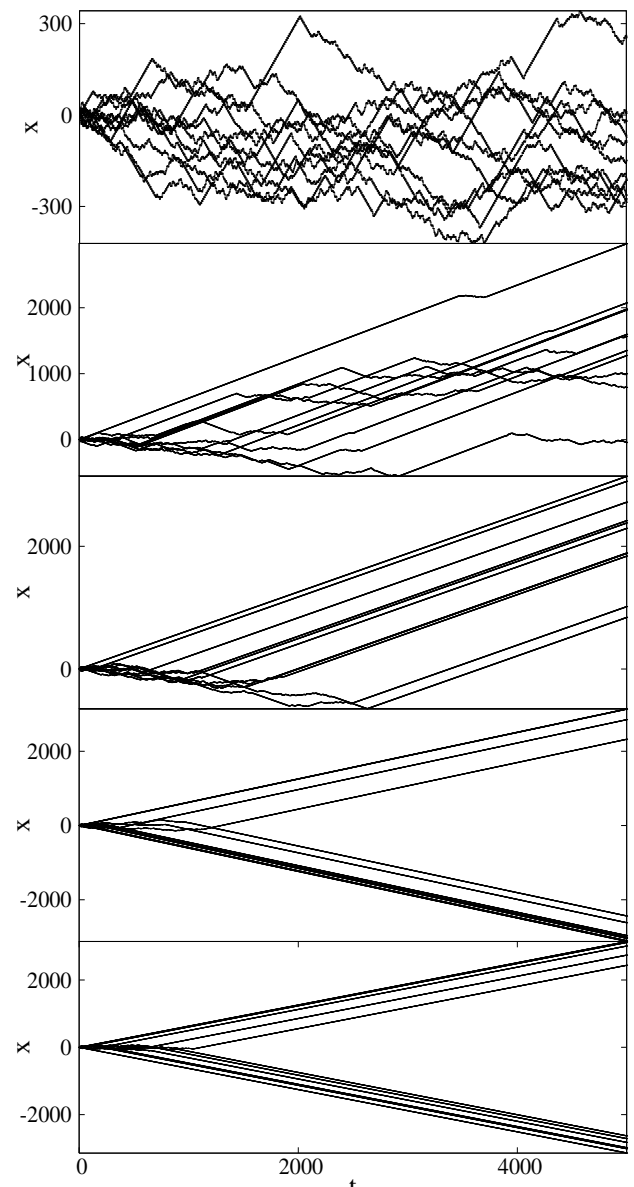

(a)

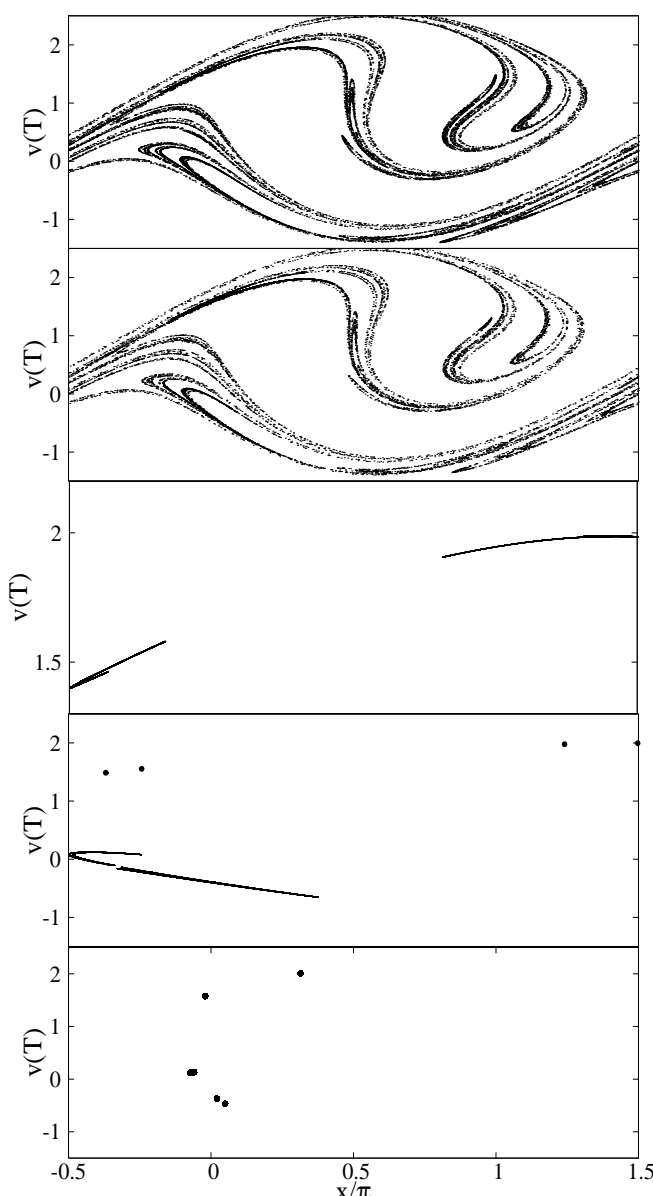

(b)

Fig. 9. Particle trajectories (a), and the corresponding stroboscopic plots (b), for different values of inhomogeneity parameter $\lambda(\lambda=0.9$ (top) to $\lambda=0.5$ (bottom). $\tau=10, a=0.67, \gamma=0.12, \phi=0.35$. Trajectories are shown for ten different sample initial conditions. Though the trajectories appear to be straight line in the scale of the figures, they are oscillatory as in Fig.3

gives a qualitative mechanism for the explanation of Fig.8 and underlines the importance of averaging over the initial phase $\phi_{0}$ at smaller inhomogeneities.

\subsection{Role of noise and low amplitudes of drives}

In deterministic ratchets, because of the complex structure of the basins of attraction and the presence of multiple attractors in phase space[38], the nature of the current is sensitively dependent on the initial conditions. The presence of noise in the system, however, makes the particle dynamics independent of the initial conditions allowing the particle to explore all the existing attractors. As a result the chaotic behaviour of the system disappears. The average velocity shows a relatively smoother variation with the amplitude of external drive than in the deterministic case. In Fig.10, the variation of $\bar{v}$ with $a$ 


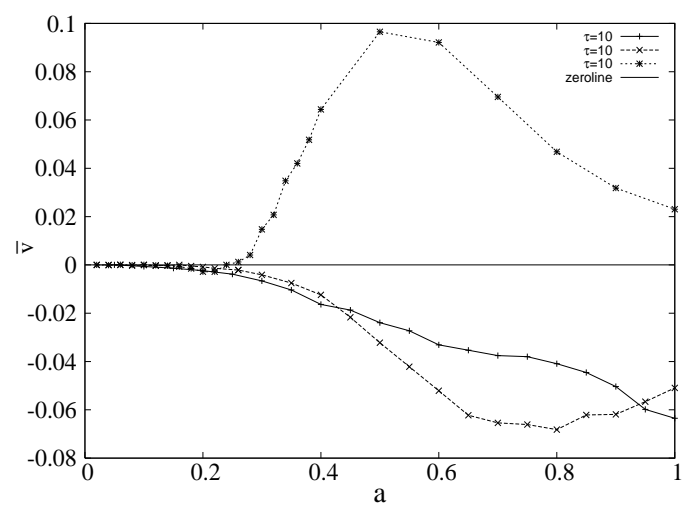

Fig. 10. Plot of $\bar{v}$ versus $a$ in the presence of noise, for three values of $\tau ; T=0.4$, $\gamma=0.12, \lambda=0.9, \phi=0.35$.

is shown for three different values of drive frequencies, keeping all the other parameters fixed.

With noise, appreciable ratchet current is obtained even at low amplitudes, where there was no current in the deterministic case (Fig.10). This is because, the presence of noise aids the particles to overcome the potential barrier which it could not have done otherwise. Hence the presence of noise plays a non-negligible role in obtaining ratchet current in the low amplitude regime, contrary to what has been mentioned by others [20]. The same effect of noise has been shown in a different system using the matrix continued fraction method [41].

\section{Conclusion}

In this work the deterministic ratchet current was obtained in a periodically driven symmetric potential in the presence of frictional inhomogeniety (most of the earlier works were with a driven asymmetric potential). The particle dynamics shows similar characteristic as in an asymmetric potential. Initial conditions and the control parameters play a major role in shaping the nature of the dynamics. For low amplitudes of drive the initial phase of the drive is found to play a non-negligible role. At these amplitudes, we show that averaging over initial conditions and initial phases of the external drive is essential to obtain realistic averages. With noise, ratchet current is obtained at amplitude regimes where there is no current in the deterministic systems.

\section{Acknowledgement}

MCM acknowledges partial finacial support from BRNS, DAE, Govt. of India under the grant No.2009/37/17/BRNS. 


\section{References}

[1] P. Reimann, Phys. Rep. 57, 361 (2002).

[2] R. D. Astumian, Science, 276, 917 (1997); R. D. Astumian and P. Hänggi, Phys. Today 55, 33 (2002).

[3] R. P. Feynmann, R. B. Leighton and M. Sands, The Feynmann Lectures in Physics, Vol.1 (Phillipines: Addison-Wesley) Chapter 46.

[4] L. Machura, M. Kostur, F. Marchesoni, P. Talkner, P. Hänggi and J. Łuczka, J. Phys. Cond. Matter 17, S3741 (2005)'

[5] A. Ajdari and J. Prost, C. R. Acad. Sci. Paris 315, 4635 (1992).

[6] R. Landaurer, J. Stat. Phys. 53, 233 (1988).

[7] M. Büttiker, Z. Phys. 68, 161 (1987); M. C. Mahato, T. P. Pareek and A. M. Jayannavar, Int. J. Mod. Phys. B 10, 3857 (1996); M. C. Mahato, Indian J. Phys. 78, 693 (2004).

[8] Ya. M. Blanter and M. Büttiker, Phys. Rev. Lett. 81, 4040 (1988).

[9] R. Benjamin and R. Kawai, Phys. Rev. E, 77, 051132 (2008).

[10] S. Saikia and M. C. Mahato, J. Phys. Cond. Matter 21, 175409 (2009); W. L. Reenbohn, S. Saikia, R. Roy and M. C. Mahato, Pramana J. Phys. 71, 297 (2008).

[11] W. L. Reenbohn and M. C. Mahato, J. Stat. Mech. P03011 (2009).

[12] M.C. Mahato and A.M. Jayannavar, Phys. Lett. A 209, 21 (1995); D.R.Chiavlo, and M.M.Millonas, Phys. Lett. A 209,26 (1995).

[13] I. Zapata, J. Łuczka, F. Sols, and P. Hänggi, Phys. Rev. Lett. 80, 829 (1998).

[14] S. Flach, O. Yevtushenko, and Y. Zolotaryuk, Phys. Rev. Lett. 84, 2358 (2000).

[15] P. Hänggi and F. Marchesoni, Rev. Mod. Phys. 81, 387 (2009).

[16] P. Jung, J. G. Kissner and P. Hänggi, Phys. Rev. Lett., 76, 3436 (1996).

[17] R. Bartussek, P. Hänggi and J. G. Kissner, Europhys Lett., 28(7), 459 (1994).

[18] J. F. Chauwin, A. Ajdari and J. Prost, Europhys Lett., 27, 421 (1994); 32, 373 (1995).

[19] A. Kenfack, S. M. Sweetnam and A. K. Pattanayak, Phys. Rev. E, 75, 056215 (2007).

[20] J. L. Mateos, Phys. Rev. Lett., 84, 258 (2000).

[21] M. Barbi and M. Salerno, Phys. Rev. E, 62, 1988 (2000). 
[22] C. M. Arizmendi, F. Family and A. L. Salas-Brito, Phys. Rev. E 63, 061104 (2001).

[23] M. Borromeo, G. Constantani and F. Marchesoni, Phys. Rev. E 65, 041110 (2002).

[24] H. A. Larrondo, F. Family and C. M. Arizmendi, Physica A 303, 67 (2002).

[25] L. Cai, Physica A, 389, 362-366 (2010).

[26] H. Risken, The Fokker-Planck Equation, Springer-Verlag, Berlin, 1989.

[27] F. Marchesoni, S. Savelev and F. Nori, Phys. Rev. E 73, 021102 (2006).

[28] M. F. Carusela, A.J. Fendrik and L. Romanelli, Physica A 388, 4017 (2009).

[29] O. Yevtushenko, S. Flach, and K. Richter, Phys. Rev. E 61, 7215 (2000).

[30] S.M. Soskin, O.M. Yevtushenko, and R. Mannella, Phys. Rev. Lett. 95, 224101 (2005).

[31] D. Hennig, L. Schimansky-Geier and P. Hänggi, Eur. Phys. J. B62, 493 (2008).

[32] M. N. Popescu, C. M. Arizmendi, A. L. Salas-Brito and F. Family, Phys. Rev. Lett, 85, 3321 (2000).

[33] H. A. Larrondo, C. M. Arizmendi and F. Family, Physica A 320, 49 (2003).

[34] F. Family, H. A. Larrondo, D. G. Zarlenga and C. M. Arizmendi, J. Phys. Cond. Matter 17, S3719, (2005).

[35] L. Gorre-Talini, J. P. Spatz, and P. Silberzan, Chaos 8, 650 (1998); I. Derenyi and R. D. Astumian, Phys. Rev. E 58, 7781, 1998; D. Ertas, Phys. Rev. Lett. 80, 1548 (1998).

[36] T. Hondou, and Y. Sawada, Phys. Rev. Lett. 75, 3269 (1995).

[37] M. Barbi and M. Salerno, Phys. Rev. E 63, 066212, (2001).

[38] J. L. Mateos, Physica A 325, 92-100, (2003).

[39] C.M. Falco, Am. J. Phys. 44, 733 (1976).

[40] A. Barone, and G. Paternó, Physics and Applications of the Josephson Effect, John Wiley \& Sons, New York, Inc., 1982.

[41] S. Denisov, P. Hänggi, and J.L. Mateos, Am. J. Phys. 77, 602606 (2009).

[42] B.A. Huberman, J.P. Crutchfield, and N.H. Packard, Appl. Phys. Lett. 37, 750 (1980); D. D'Humieres, M.R. Beasley, B.A. Huberman, and A. Libchaber, Phys. Rev. A 26, 3483 (1982). 